\title{
Integrated Flood Risk Assessment System Based on the Set Pair Analysis and Variable Fuzzy Sets for Cascade Dams
}

\author{
Yu CHEN $\mathrm{a}$,b,1 \\ ${ }^{a}$ State Key Laboratory of Hydraulics and Mountain River Engineering, Sichuan \\ University, Chengdu 610065, China \\ ${ }^{b}$ Institute for Risk and Disaster Reduction, University College London, Gower Street, \\ London WC1E 6BT, United Kingdom
}

\begin{abstract}
Flood-caused dam break is an important dam safety problem. Dam flood risk assessment based on an appropriate assessing method is hence essential for reducing dam-break flood damages and losses. In this research, a comprehensive flood risk assessment system for cascade dams was developed by combining the set pair analysis (SPA) and variable fuzzy sets (VFS) theory (namely SPA-VFS for short), and expressing the flood risk as a product of hazard and vulnerability, which were quantified by selected indicators. In the case study of the Dadu river basin, reservoir capacity, dam height, dam age, gross domestic product (GDP) density, population density, and predicted damage were selected as the evaluation indicators, and the evaluation results derived the flood risk classes of each studied dam. The study indicates that the proposed SPA-VFS-based flood risk assessment model is simple and feasible, which enables its further application in flood risk analysis and evaluation, and its extension in the risk assessment of other natural disasters.
\end{abstract}

Keywords. Flood, set pair analysis (SPA), variable fuzzy sets (VFS), risk assessment, cascade dams

\section{Introduction}

Flood is one in every of the foremost cosmopolitan natural disasters to life and property in the world. The dam break caused by flood often brings catastrophic damages and enormous impacts to humans, society, economy, and environment, and becomes a worldwide problem. The gradually increasing flood occurrence provokes the development of dam safety management and flood risk decision-making, which is closely based on high-accuracy dam flood risk assessment.

Many approaches, such as uncertainty analysis, statistical analysis, and multi-criteria decision-making analysis, have been applied in risk assessment [1]. In recent years, more and more researchers pay attention to the uncertainty method, such as the variable fuzzy sets (VFS) theory and set pair analysis (SPA) method. In this context, an integrated flood

\footnotetext{
${ }^{1}$ Corresponding Author: Yu Chen, State Key Laboratory of Hydraulics and Mountain River Engineering, Sichuan University, Chengdu 610065, China; E-mail: rainchen393@hotmail.com

This work was supported by Sichuan Science and Technology Program [grant number 2019YFH0140]; International Visiting Program for Excellent Young Scholars of SCU; and the National Natural Science Foundation of China [grant numbers 41501554].
} 
risk system combining SPA and VFS methods was constructed and implemented for cascade dams in the study area.

\section{Methodology}

\subsection{Variable Fuzzy Sets (VFS)}

The VFS theory based on the relative difference function is a convenient tool for processing random, fuzzy, and multi-indicator problems, and has been used in the field of risk evaluation. Denote the amount of sample sets by $n$, then

$$
X=\left\{x_{1}, x_{2}, \ldots, x_{n}\right\}
$$

where $x$ is the sample set.

Let there are $m$ indicators, the indicator eigenvalue of $j$ th sample is represented as

$$
x_{j}=\left\{x_{1 j}, x_{2 j}, \ldots, x_{m j}\right\}
$$

Based on $i$ th $(i=1,2, \ldots, m)$ indicator eigenvalue of $j$ th $(j=1,2, \ldots, n)$ sample, $x_{i j}$, , the sample set can be defined as follows.

$$
X=\left(x_{i j}\right)
$$

To define VFS, let $u$ is the arbitrary element and $U$ is a fuzzy concept set, for $\forall u \in U, \mu_{A}(u), 0 \leq \mu_{A}(u) \leq 1$ and $\mu_{A^{c}}(u), 0 \leq \mu_{A^{c}}(u) \leq 1$ are relative membership degrees of $u$ to the attractive intervals $A$ and $A^{c}$, respectively, describing attractability and repellency respectively, and $\mu_{A}(u)+\mu_{A^{c}}(u)=1$. Define $V$ as VFS, $V=\left\{\left(u, D_{A}(u)\right) \mid u \in U, D_{A}(u)=\mu_{A}(u)-\mu_{A^{c}}(u), D_{A}(u) \in[-1,1]\right\}$ as VFS, where $D_{A}(u)$ is the relative difference degree of $u$ to $A$. Furthermore, suppose $A_{+}, A_{-}$, and $A_{0}$ are attracting, repelling and qualitative change sets of $V$, respectively [2], then, $A_{+}=\left\{u \mid u \in U, D_{A}(u) \in(0,1)\right\} \quad, \quad A_{-}=\left\{u \mid u \in U, D_{A}(u) \in(-1,0)\right\} \quad, \quad$ and $A_{0}=\left\{u \mid u \in U, D_{A}(u)=0\right\}$.

Considering the advantage of VFS for expressing the nonlinear relationship between evaluation indicators and risk grades, and the limitation of VFS for solving assessment indicators induced information duplication, more and more theories and methods are used to combine with VFS for various improvements.

\subsection{Set Pair Analysis (SPA)}

SPA is a new theory of expressing and tackling the systematic uncertainty by integrating uncertainty and certainty as to the certain-uncertain system, dividing the certainuncertain relationship into identity, contrary and discrepant, and utilizing a connection number to represent degrees of identity, contrary and discrepancy in specific cases based on analyzing characteristics of the studied set pair. SPA is used in this paper to calculate the relative membership degree. Let $H(A, B)$ is a set pair formed by putting together two 
sets $A$ and $B$, the connection degree $\mu_{(A-B)}$ of the set pair can be expressed as the following three-element connection form [3-4]:

$$
\mu_{(A-B)}=a+b i+c j
$$

The general form of connection degree shown in Eq. (4) can be extended to the fiveelement connection degree as follows [5]:

$$
\mu_{(A-B)}=a+b i+c j=a+b_{1} i_{1}+b_{2} i_{2}+b_{3} i_{3}+c j
$$

where $a+b_{1}+b_{2}+b_{3}+c=1, a ; b_{1}, b_{2}, b_{3} ; c$ are components of identity degree; discrepancy degree; and contrary degree, respectively, and used to determine the grades of evaluation indicators in flood risk assessment; $i_{1}, i_{2}, i_{3}$ are uncertainty coefficients of discrepancy degree and $i_{1}, i_{2}, i_{3} \in[-1,1] ; j=-1$.

\subsection{Flood Risk Assessment Based on SPA-VFS}

The VFS based on the membership function is limited by complicated calculation and multi-variables requirements for deriving the discrepancy degree. SPA provides a new and simple way to establish the connection degree, which is basically the same as the discrepancy degree. The two methods hence can be combined to form a SPA-VFS flood risk assessment system, which can be implemented in the following steps [6]:

(1) Determine assessment indicators and standards of the dam flood risk. Denote the assessment sample as $\left\{x_{j} \mid j=1,2, \ldots, m\right\}$ and corresponding evaluation grade standard as $\left\{s_{j k} \mid j=1,2, \ldots, m ; k=1,2, \ldots, n\right\}$, where $m, n$ are the total numbers of indicators and grades, respectively. $x_{j}$ is the eigenvalue of the indicator $j, s_{j k}$ is the evaluation standard for the indicator $j$ and level $k$.

(2) Calculate the indicator weights $\left\{w_{j} \mid j=1,2, \ldots, m, \sum_{j=1}^{m} w_{j}=1\right\}$ by using the analytic hierarchy process (AHP) method, where $w_{j} \in[0,1]$ is the weight of the indicator $j$. More details about AHP can reference the research of Orencio and Fujii [7].

(3) Derive the connection degree between two sets of evaluation indicators and assessment levels by using SPA. If the evaluation indicators of samples $1,2, \ldots, l_{l}$ are in the grade $k$, then the identity degree $a$ is defined as $a=\sum_{j=1}^{l_{1}} w_{j}$. If the evaluation indicators of samples $l_{1}+1, l_{1}+2, \ldots, l_{2}$ are located in the grade $k$ interval, the contrary degree $c$ is denoted as $c=\sum_{j=l_{1}+1}^{l_{2}} w_{j}$. If the evaluation indicators of samples $l_{2}+1$, $l_{2}+2, \ldots, m$ are located in the grade $k$ adjacent, the discrepancy degree $b_{j}$ of a single indicator is expressed as $b_{j}=w_{j},\left(j=l_{2}+1, l_{2}+2, \ldots, m\right)$. And the difference degree coefficient $I_{j}$ for a single indicator is 


$$
I_{j}=\left\{\begin{array}{cc}
1-2 \cdot\left|\frac{s_{j(k-1)}-x_{j}}{s_{j(k-1)}-s_{j(k-2)}}\right| & x_{j} \in \operatorname{grade}(k-1) \\
1-2 \cdot\left|\frac{x_{j}-s_{j k}}{s_{j(k+1)}-s_{j k}}\right| & x_{j} \in \operatorname{grade}(k+1)
\end{array}\right.
$$

where $x_{j} \in$ grade $(k-1)$ and $x_{j} \in$ grade $(k+1)$ represent the indicator values locate in the grade $k-1$ adjacent and grade $k+1$ adjacent, respectively.

The connection degree $u_{j}$ between $x_{j}$ and the grades $k$ is

$$
u_{j}=a+\sum_{j=l_{2}+1}^{m} b_{j} \cdot I_{j}+c \cdot J
$$

where $J=-1$.

Then the synthetic connection degree $u$ can be calculated as [4]:

$$
u=\sum_{j=1}^{m} w_{j} \cdot u_{j}
$$

where $u \in[-1,1]$.

(4) Calculate the relative membership degree $v_{k}$ based on the relation degree $u_{k}$ of grade $k$.

$$
v_{k}=0.5+0.5 \cdot u_{k}
$$

(5) Calculate the assessment grade feature value $H$ by using the rank feature value method [8]:

$$
H=\sum_{k=1}^{n} k \cdot\left(v_{k} / \sum_{k=1}^{n} v_{k}\right)
$$

In this study, flood risk grades are illustrated as lowest, low, moderate, high, and highest, denoted by 1, 2, 3, 4, and 5, respectively. Based on the calculation results of Eq. (10), the qualitative grades can be assigned to each dam according to the following rule [9]:

$$
\text { risk grade }= \begin{cases}1, & 1.0 \leq H \leq 1.5 \\ 2, & 1.5<H \leq 2.5 \\ 3, & 2.5<H \leq 3.5 \\ 4, & 3.5<H \leq 4.5 \\ 5, & 4.5<H \leq 5.0\end{cases}
$$

\section{Case study}

The Dadu river basin is located in the southwest of China, where one of the thirteen hydropower bases is distributed. Currently, there are a total of 22 planned, underconstructed, and built cascade reservoirs in the mainstream, wherein Shuangjiangkou and Pubugou dams are the control hydraulic projects of the cascade reservoir system, and situated in the upstream and the downstream of the basin, respectively. From upstream to downstream, Busigou (BSG), Shuangjiangkou (SJK), Luding (LD), 
Dagangshan (DGS), Longtoushi (LTS), and Pubugou (PBG) dams are selected as study examples, and each dam is regarded as a basic assessment unit.

According to the disaster risk theory, the flood risk can be expressed as a product of flood hazard and flood vulnerability. Correspondingly, the studied flood risk assessment indicators should be selected from the aspects of disaster-inducing factors, disasterforming environment, and disaster-bearing bodies. Considering engineering properties of cascade dams and the natural quality of floods, six indicators are chosen, including three hazard indicators of reservoir capacity $\left(\mathrm{C}_{1}\right)$, dam height $\left(\mathrm{C}_{2}\right)$ and dam age $\left(\mathrm{C}_{3}\right)$, and three vulnerability indicators of GDP density $\left(\mathrm{C}_{4}\right)$, population density $\left(\mathrm{C}_{5}\right)$, and predicted damage $\left(\mathrm{C}_{6}\right)$. It should be noted that $\mathrm{C}_{6}$ is a qualitative indicator, and should be characterized by the highest water level of the reservoir (HWL).

To quantify these risk indicators, the historical flood information, statistical yearbook, field survey data, and expert judgments are collected. After that, each indicator is divided into five levels from lowest (1) to highest (5), and the division is shown in Table 1.

The indicator weights are calculated by the AHP method as:

$$
w_{1}=(0.190,0.111,0.032,0.059,0.130,0.478)
$$

It can be seen from the weighting result that the predicted damage $\left(\mathrm{C}_{6}\right)$ indicator with the highest weight is the most important risk influencing factor, while the dam age $\left(\mathrm{C}_{3}\right)$ indicator is the least important one.

After obtaining the indicator weights, Eqs. (6)-(10) was applied step by step to calculate the single and synthetic connection degrees, the relative and normalized membership degrees, and the assessment grade feature values. The final risk grade of each dam was determined in the light of Eq. (11) and shown in Table 2.

The results show that dams located in the upper reaches possess low risk, while dams distributed in the middle reaches possess moderate risk. Low-risk dam locations are relatively low-developed economic and sparse population distribution areas. For midstream areas with high GDP and population density and an intense rainfall zone, where the safety performance of the dams is worse than that of upstream dams, which is reflected by the relatively higher grade feature values and risk grade ratings. Specifically, the dam height of the DGS dam is more than $200 \mathrm{~m}$, and the reservoir capacity of the PBG dam is more than 5 billion $\mathrm{m}^{3}$. The SJK dam is a control project of the basin cascade system, plays the role of energy production and flood prevention, and is the most sensitive cascade because its failure will induce catastrophic damage for the downstream cascade reservoirs.

Table 1. Level standards of flood risk assessment indicators.

\begin{tabular}{llllll}
\hline Risk indicators & Lowest & Low & Moderate & High & Highest \\
\hline $\begin{array}{l}\text { Reservoir capacity } \\
\left(10^{6} \mathrm{~m}^{3}\right)\end{array}$ & $<1$ & $1-10$ & $10-100$ & $100-1000$ & $>1000$ \\
Dam height $(\mathrm{m})$ & $<15$ & $15-70$ & $70-166$ & $166-300$ & $>300$ \\
Dam age (years) & $<5$ & $5-45$ & $45-85$ & $85-115$ & $>115$ \\
$\begin{array}{l}\text { GDP density } \\
\left(\text { billion yuan } / \mathrm{km}^{2}\right)\end{array}$ & $<10$ & $10-40$ & $40-80$ & $80-100$ & $>100$
\end{tabular}




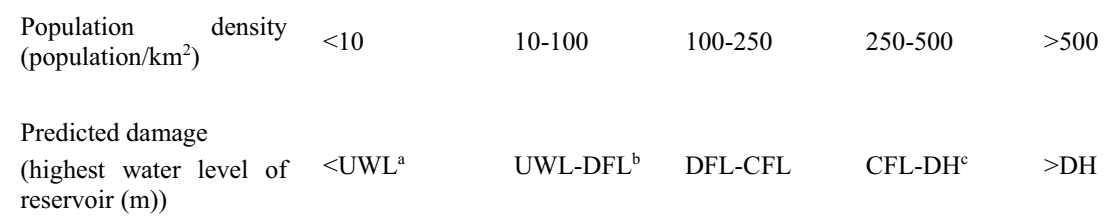

${ }^{\mathrm{a}}$ UWL-Upper water level for flood control;

${ }^{\mathrm{b}}$ DFL-Design flood level;

${ }^{\mathrm{c}}$ CFL-Checked flood level; DH-Dam height.

Table 2. Results of the flood risk grades of the studied dams.

\begin{tabular}{lllllll}
\hline $\begin{array}{l}\text { Normalized } \\
\text { membership }\end{array}$ & BSG & SJK & LD & DGS & LTS & PBG \\
\hline 1 & 0.190 & 0.521 & 0.239 & 0.062 & 0.127 & 0.058 \\
2 & 0.438 & 0.156 & 0.318 & 0.188 & 0.246 & 0.159 \\
3 & 0.334 & 0.140 & 0.112 & 0.304 & 0.381 & 0.427 \\
4 & 0.038 & 0.100 & 0.011 & 0.328 & 0.246 & 0.341 \\
5 & 0.000 & 0.083 & 0.320 & 0.118 & 0.000 & 0.015 \\
Grade feature value & 2.2 & 2.068 & 2.855 & 3.252 & 2.746 & 3.096 \\
Risk grades & Low & Low & Moderate & Moderate & Moderate & Moderate \\
\hline
\end{tabular}

\section{Conclusions}

This study takes cascade dams, BSG, SJK, LD, DGS, LTS, and PBG, in the Dadu river basin as an example to conduct an integrated flood risk assessment. According to the disaster risk theory and actual situation of the study case, three hazard indicators and three vulnerability indicators are selected and then weighted by the AHP method. The integrated flood risk assessment of each studied dam was implemented by the built SPAVFS model, and the resulting flood risk grades of each dam indicated that the cascade dams are safe. The SPA-VFS retains the advantages of SPA and VFS methods, can simplify the calculation of the discrepancy degree using SPA and identify the membership relationship between assessment indicators and standards utilizing VFS theory, and overcome the limitations of the maximum membership degree principle (MMDP) in traditional SPA method.

\section{References}

[1] Jiang WG, Deng L, Chen LY, Wu JJ and Li J. Risk assessment and validation of flood disaster based on fuzzy mathematics. Progress in Natural Science. 2009; 19:1419-1425.

[2] Chen SY, Guo Y. Variable fuzzy sets and its application in comprehensive risk evaluation for floodcontrol engineering system. Fuzzy Optim Decis Mak. 2006; 5:153-162.

[3] Zou Q, Zhou JZ, Zhou C, Song LX and Guo J. Comprehensive flood risk assessment based on set pair analysis-variable fuzzy sets model and fuzzy AHP. Stochastic Environmental Research Risk Assessment. 2013; 27: 525-546.

[4] Guo EL, Zhang JQ, Ren XH, Zhang Q and Sun ZY. Integrated risk assessment of flood disaster based on improved set pair analysis and the variable fuzzy set theory in central Liaoning Province, China. Natural 
Hazards. 2014; 74:947-965.

[5] Chen Y and Shu LC. Set pair analysis for regional land subsidence vulnerability assessment. 2012 International Conf. on Computer and Information Science, Safety Engineering (CAISSE 2012), June 1517, 2012, Wuhan.

[6] Zhou JF. SPA-fuzzy method based real-time risk assessment for major hazard installations storing flammable gas. Safety Science. 2010; 48:819-822.

[7] Orencio PM and Fujii M. A localized disaster-resilience index to assess coastal communities based on an analytic hierarchy process (AHP). International Journal of Disaster Risk Reduction. 2012; 3:62-75.

[8] Chen SY and Guo Y. Variable fuzzy sets and its application in comprehensive risk evaluation for floodcontrol engineering system. Fuzzy Optimization and Decision Making. 2006; 5:153-162.

[9] Guo Y 2006 Research on application of engineering fuzzy sets theory in water resources and flood control system. Dalian University of Technology, Dalian, 2006. 